\title{
Methotrexate administration directly into the fourth ventricle in children with malignant fourth ventricular brain tumors: a pilot clinical trial
}

\author{
David I. Sandberg ${ }^{1,2} \cdot$ Michael Rytting ${ }^{3} \cdot$ Wafik Zaky $^{3} \cdot$ Marcia Kerr $^{4}$ • \\ Leena Ketonen ${ }^{5}$ - Uma Kundu ${ }^{6}$ - Bartlett D. Moore ${ }^{3}$ - Grace Yang ${ }^{3}$. \\ Ping Hou ${ }^{7}$ - Clark Sitton ${ }^{8}$ - Laurence J. Cooper ${ }^{3,9}$ • Vidya Gopalakrishnan ${ }^{3}$. \\ Dean A. Lee $^{3} \cdot$ Peter F. Thall ${ }^{10} \cdot$ Soumen Khatua ${ }^{3}$
}

Received: 14 April 2015 / Accepted: 3 August 2015/Published online: 9 August 2015

(C) The Author(s) 2015. This article is published with open access at Springerlink.com

\begin{abstract}
We hypothesize that chemotherapy can be safely administered directly into the fourth ventricle to treat recurrent malignant brain tumors in children. For the first time in humans, methotrexate was infused into the fourth ventricle in children with recurrent, malignant brain tumors. A catheter was surgically placed into the fourth ventricle and attached to a ventricular access device. Cerebrospinal fluid (CSF) flow was confirmed by CINE MRI postoperatively. Each cycle consisted of 4 consecutive daily methotrexate infusions ( 2 milligrams). Disease response was monitored with serial MRI scans and CSF cytologic analysis. Trough CSF methotrexate levels were sampled. Five patients ( 3 with medulloblastoma and 2 with ependymoma) received 18, 18, 12, 9, and 3 cycles, respectively. There were no serious adverse events or new neurological deficits attributed to methotrexate. Two
\end{abstract}

David I. Sandberg

david.i.sandberg@uth.tmc.edu

1 Division of Pediatric Neurosurgery, Departments of Pediatric Surgery and Neurosurgery, University of Texas Health Science Center at Houston and Mischer Neuroscience Center, 6431 Fannin Street, MSB 5.144, Houston, TX 77030, USA

2 Divisions of Neurosurgery and Pediatrics, University of Texas MD Anderson Cancer Center, Houston, TX, USA

3 Division of Pediatrics, Unit 87, University of Texas MD Anderson Cancer Center, 1515 Holcombe Blvd., Houston, TX 77030, USA

4 Division of Neurosurgery, Department of Pediatric Surgery, University of Texas Health Science Center at Houston, 6431 Fannin St., MSB 5.146, Houston, TX 77030, USA

5 Unit 1482, Department of Diagnostic Imaging, Section of Neuroradiology, FCT 16.5020, University of Texas MD Anderson Cancer Center, 1400 Pressler Street, Houston, TX 77030, USA additional enrolled patients were withdrawn prior to planned infusions due to rapid disease progression. Median serum methotrexate level $4 \mathrm{~h}$ after infusion was $0.04 \mu \mathrm{mol} /$ L. Range was $0.02-0.13 \mu \mathrm{mol} / \mathrm{L}$. Median trough $\mathrm{CSF}$ methotrexate level $24 \mathrm{~h}$ after infusion was $3.18 \mu \mathrm{mol} / \mathrm{L}$ (range $0.53-212.36 \mu \mathrm{mol} / \mathrm{L}$ ). All three patients with medulloblastoma had partial response or stable disease until one patient had progressive disease after cycle 18 . Both patients with ependymoma had progressive disease after 9 and 3 cycles, respectively. Low-dose methotrexate can be infused into the fourth ventricle without causing neurological toxicity. Some patients with recurrent medulloblastoma experience a beneficial anti-tumor effect both within the fourth ventricle and at distant sites.

6 Unit 85, Department of Pathology, University of Texas MD Anderson Cancer Center, 1515 Holcombe Blvd., Houston, TX 77030, USA

7 Unit 1472, Department of Imaging Physics, University of Texas MD Anderson Cancer Center, 1515 Holcombe Blvd., Houston, TX 77030, USA

8 Department of Diagnostic \& Interventional Imaging, University of Texas Health Science Center at Houston, 6431 Fannin Street, MSB 2.130B, Houston, TX 77030, USA

9 Ziopharm Oncology Inc., 1 First Avenue; Parris Building, \#34, Navy Yard Plaza, Boston, MA 02129, USA

10 Department of Biostatistics, Office FCT 4.614, MD Anderson Cancer Center, Houston, TX 77230-1402, USA 
Keywords Fourth ventricle $\cdot$ Intraventricular chemotherapy $\cdot$ Medulloblastoma $\cdot$ Ependymoma . Methotrexate

\section{Introduction}

Despite improved outcomes for patients with medulloblastoma and other malignant posterior fossa tumors over the past few decades, survival rates remain low for patients with recurrence following initial surgical resection and adjuvant therapy [1]. Moreover, current treatment regimens for relapsed malignancies can cause considerable morbidity. Novel approaches are warranted in order to improve survival rates and minimize toxicity. This pilot clinical trial is based upon the hypothesis that infusion of chemotherapeutic agents directly into the fourth ventricle of the brain may optimize local and regional drug concentrations and improve tumor control rates.

This study marks the first trial in humans of chemotherapy administration into the fourth ventricle. Our primary objective was to demonstrate that chemotherapy may be safely infused into the fourth ventricle without causing new neurological deficits or evidence of damage to the adjacent brainstem or cerebellum on magnetic resonance imaging (MRI) scans. Our secondary objective was to assess if methotrexate infusions into the fourth ventricle, without any simultaneous systemic chemotherapy, had any measurable disease response.

\section{Materials and methods}

This study (clinicaltrials.gov ID NCT01737671) was performed in accordance with the ethical standards as outlined in the 1964 Declaration of Helsinki and its later amendments. The study was initiated after IND exemption was obtained from the FDA (IND Exemption Number 116804) and institutional review board approval was granted by the University of Texas MD Anderson Cancer Center (Protocol 2012-0823) and Children's Memorial Hermann Hospital/ University of Texas Health Science Center at Houston (Protocol HSC-MS-12-0492). All patients and/or their parents or legal guardians signed informed consent.

Eligible patients included children $\leq 21$ years of age with medulloblastoma, ependymoma, or atypical teratoid/ rhabdoid tumor (ATRT) that originated in the posterior fossa and subsequently recurred anywhere in the brain and/ or spine. Patients were excluded if they were pregnant, receiving any other chemotherapy, or enrolled in another experimental protocol. An estimated life expectancy of at least 12 weeks and a Karnofsky or Lansky performance score of 50 or greater was required [2].
Enrolled patients underwent a posterior fossa craniotomy and maximal safe surgical resection if resectable tumor was within the operative field. A ventricular catheter was then placed under direct vision into the fourth ventricle. The dura mater was closed in a water-tight fashion around the catheter, and the catheter was attached to a ventricular access device (VAD) which was implanted subcutaneously at the inferior aspect of the incision (Fig. 1).

Postoperatively, patients underwent an MRI of the brain with gadolinium to assess both baseline tumor burden and catheter placement within the fourth ventricle (Fig. 2). Additionally, CINE MRI sequences of the brain and total spine were performed to confirm CSF flow from the fourth ventricular outlets to the cervical, thoracic, and lumbar spine. CSF flow was characterized as present or absent by the study neuroradiologist. If CSF flow from the fourth ventricle to the lumbar spine was not definitively present, then a nuclear medicine CSF flow study with Indium-111 diethylenetriaminepenta-acetic acid (In-111 DPTA) was required prior to methotrexate infusions. Patients additionally underwent lumbar puncture to assess CSF cytology at least 10 days postoperatively and before initiation of chemotherapy.

Intraventricular methotrexate cycles were initiated at least 2 weeks after VAD placement. All patients were admitted to the hospital for the first 3 cycles, and subsequent cycles were performed on an outpatient basis. Neurological examinations were performed immediately before and after each infusion. Each cycle consisted of four consecutive daily infusions of methotrexate $(2 \mathrm{mg}$ prepared in $2 \mathrm{~mL}$ of preservative-free normal saline followed by a $1 \mathrm{~mL}$ flush of preservative-free normal saline). Prior to each infusion, $3 \mathrm{~mL}$ of CSF was aspirated from the VAD for trough

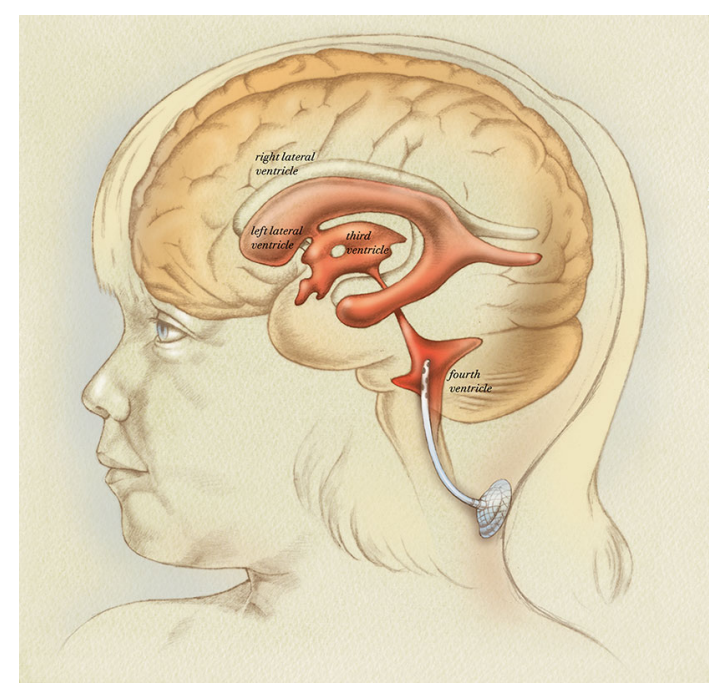

Fig. 1 Artist's illustration demonstrating surgical placement of catheter into the fourth ventricle of the brain attached to subcutaneous reservoir 

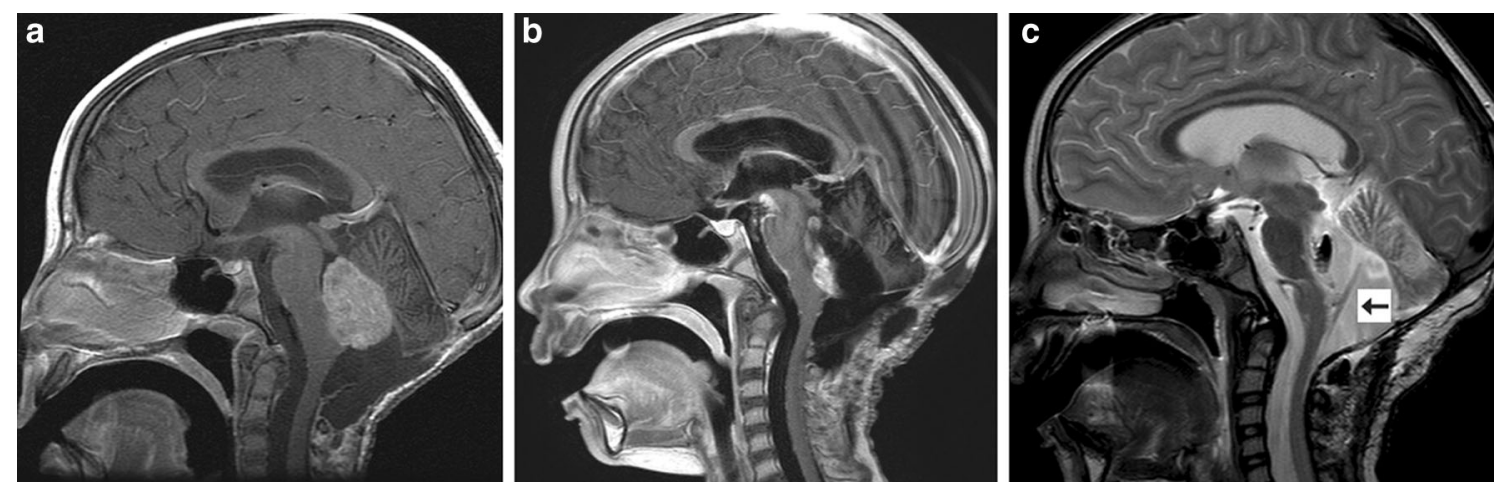

Fig. 2 Preoperative and postoperative MRI images from patient 2. a Preoperative sagittal T1-weighted MRI with gadolinium demonstrating recurrent, enhancing tumor filling fourth ventricle. b Postoperative sagittal T1-weighted MRI with gadolinium demonstrating subtotal resection of tumor. A small amount of tumor adherent to the floor of the fourth ventricle was purposefully left behind.

methotrexate level, cytology, gram stain and culture, cell count, glucose and protein. Serum methotrexate levels were measured $4 \mathrm{~h}$ after each methotrexate infusion for at least the first 3 cycles. Serum and CSF methotrexate levels were measured using previously reported fluorescence polarization immunoassay techniques [3]. Leucovorin rescue (5 mg/ $\mathrm{m}^{2}$ per dose) was planned if any serum methotrexate level was $>0.3 \mu \mathrm{mol} / \mathrm{L}$. Patients did not receive any simultaneous systemic chemotherapy.

An MRI scan of the brain was obtained within $48 \mathrm{~h}$ after completion of the first cycle primarily to identify new signal changes in the brain after methotrexate infusions. MRI scans of the brain and/or spine to assess disease response were obtained after the third cycle and after every third cycle thereafter until therapy was stopped. Disease response was assessed by comparing MRI scans after surgery (pre-treatment baseline) with MRI scans obtained after every subsequent three cycles. Lumbar puncture to assess CSF cytology was repeated after the third cycle and subsequently after every additional three cycles if the prior lumbar puncture showed positive cytology. Table 1 outlines criteria used for assessing disease response. Criteria for stopping therapy included either disease progression or the judgment of the patient's treating physicians that maximal benefit had been achieved. All patients underwent neuropsychological evaluation with age-appropriate test batteries postoperatively before fourth ventricular chemotherapy administration and again after completion of the third cycle.

\section{Results}

Seven patients with a median age of 12 years (range 4-19 years) were enrolled (Table 2). Five patients had recurrent medulloblastoma, and two had recurrent c Postoperative T2-weighted sagittal MRI demonstrating catheter position within the fourth ventricle. The catheter, as shown by the arrow, is positioned in a trajectory such that injury to the brainstem and cerebellum are avoided and all catheter holes are within the fourth ventricle

anaplastic ependymoma. All seven patients had progressive disease at the time of enrollment despite prior surgery, radiation therapy, and various chemotherapy regimens. Two patients (patients 1 and 3) had received prior intravenous methotrexate, both at least 3 years prior to enrollment.

All patients underwent successful surgical implantation of the catheter and VAD. Simultaneously, three patients underwent subtotal resection of tumor located in the fourth ventricle or cerebellum, and one patient underwent simultaneous tumor biopsy. The remaining three patients did not have disease in the posterior fossa amenable to simultaneous resection. Six patients had no new neurological deficits after surgery, and one patient (patient 7) had partial left-sided facial weakness postoperatively. This patient had undergone simultaneous subtotal resection of tumor (ependymoma) that was filling the fourth ventricle. The post-operative seventh nerve palsy was attributed to resection of tumor close to the floor of the fourth ventricle and did not resolve for the duration of follow-up. Postoperative MRI scans in all patients demonstrated catheter placement in the fourth ventricle without injury to the brainstem or cerebellum (Fig. 2). In all patients, CINE MRI sequences confirmed CSF flow from the fourth ventricular outlets to the cervical, thoracic, and lumbar spinal subarachnoid spaces. Nuclear medicine CSF flow studies were therefore not required in any patients.

Two patients (patients 3 and 6) underwent surgery for VAD implantation but never received methotrexate infusions. Both of these patients were discharged from the hospital postoperatively with no new neurological deficits. In the interval between surgery and the planned first cycle, both patients had rapid disease progression. Patient 3 presented with irritability and decreased strength in his arms and legs 2 weeks postoperatively. A new MRI scan 
Table 1 Assessment of disease response

\section{Selection of target and non-target lesions}

Target lesions All measurable lesions were defined as "target" lesions. If multiple measurable lesions were present, up to five were selected as target lesions based upon size and suitability for accurate repeated measurements. Lesions were measured in whichever MRI sequence (i.e. T1, T2, FLAIR, etc.) which provided the most accurate measurement of tumor size

Non-target lesions Included lesions which could not be measured accurately, such as leptomeningeal disease. CSF cytology (e.g. CSF positive or negative for tumor cells) was followed as a non-target lesion

\section{Response criteria for target lesions}

Target lesions were measured in 2 dimensions: the longest diameter and perpendicular to the longest diameter. Response criteria were assessed based on the product of the longest diameter and its longest perpendicular diameter

Complete response $(C R)$ No evidence of disease

Partial response $(P R) \geq 50 \%$ decrease in the sum of the products of the two perpendicular diameters of all target lesions (up to 5)

Stable disease $(S D)$ Neither sufficient decrease in sum of the products of the two perpendicular diameters of all target lesions to qualify for PR nor sufficient increase in a single target lesion to qualify for PD

Progressive disease $(P D) \geq 25 \%$ increase in the product of perpendicular diameters of any target lesion or the appearance of any new lesions

\section{$\underline{\text { Response criteria for non-target lesions }}$}

Complete response $(C R)$ Disappearance of all non-target lesions

Stable disease (SD)/incomplete response (IR) The persistence of one or more non-target lesions

Progressive disease $(P D)$ The appearance of one or more new lesions and/or unequivocal progression of existing non-target lesions

\begin{tabular}{|c|c|c|c|}
\hline \multicolumn{4}{|c|}{ Overall response assessment } \\
\hline Target lesions & $\begin{array}{l}\text { Non-target } \\
\text { lesions }\end{array}$ & $\begin{array}{l}\text { New } \\
\text { lesions }\end{array}$ & $\begin{array}{l}\text { Overall } \\
\text { response }\end{array}$ \\
\hline $\mathrm{CR}$ & $\mathrm{CR}$ & No & $\mathbf{C R}$ \\
\hline $\mathrm{CR}$ & IR/SD & No & PR \\
\hline PR & $\mathrm{CR}, \mathrm{IR} / \mathrm{SD}$ & No & $\mathbf{P R}$ \\
\hline SD & $\mathrm{CR}, \mathrm{IR} / \mathrm{SD}$ & No & SD \\
\hline PD & Any & Yes or no & PD \\
\hline Any & PD & Yes or no & PD \\
\hline Any & Any & Yes & PD \\
\hline
\end{tabular}

Overall Response Assesment values are in bold

Table 2 Patient data and treatment responses

\begin{tabular}{|c|c|c|c|c|c|c|}
\hline Patient $^{\mathrm{a}}$ & Age/sex & Diagnosis & $\begin{array}{l}\text { Recurrent disease sites at time of } \\
\text { enrollment }\end{array}$ & $\begin{array}{l}\text { Extent of } \\
\text { resection }\end{array}$ & $\begin{array}{l}\text { \# of } \\
\text { cycles }\end{array}$ & $\begin{array}{l}\text { Treatment } \\
\text { response }^{c}\end{array}$ \\
\hline 1 & $19 / \mathrm{M}$ & Medulloblastoma & $\begin{array}{l}\text { 4th ventricle, cerebellar folia, } \\
\text { posterior fossa cisterns, } \\
\text { supratentorial, spine }\end{array}$ & Biopsy & 18 & SD \\
\hline 2 & $9 / \mathrm{M}$ & Anaplastic Ependymoma & $\begin{array}{l}\text { 4th ventricle, leptomeninges of } \\
\text { brainstem and cervical spine }\end{array}$ & Subtotal resection & 9 & SD \\
\hline 4 & $12 / \mathrm{M}$ & Medulloblastoma & Spinal subarachnoid space & None & 12 & PR \\
\hline 5 & $16 / \mathrm{F}$ & Medulloblastoma & Right and Left lateral ventricles & None & 18 & PR \\
\hline 7 & $8 / \mathrm{M}$ & Anaplastic Ependymoma & 4th ventricle & Subtotal resection & 3 & PD \\
\hline
\end{tabular}

$S D$ Stable disease, $P R$ partial response, $P D$ progressive disease, $N / A$ not applicable

a Patients 3 and 6 are not included in this Table because they were enrolled and underwent fourth ventricle catheter/reservoir placement but never received treatment

${ }^{\mathrm{b}}$ In addition to fourth ventricle catheter/reservoir placement

${ }^{c}$ Best treatment response

demonstrated massive hydrocephalus caused by widespread worsening of leptomeningeal tumor throughout the brain as well as tumor progression in the spine causing spinal cord compression. A palliative ventriculoperitoneal shunt was placed, and he was taken off study without ever receiving methotrexate infusions. He died 10 days later. 
Similarly, patient 6 presented 7 days postoperatively with swallowing difficulty and facial asymmetry followed by vomiting and lethargy. An MRI demonstrated massive tumor progression throughout the brain. She was taken off study and died 2 days later.

The five patients who underwent methotrexate infusions received $18,18,12,9$, and 3 cycles respectively, totaling 240 infusions. There were no new neurological deficits after these infusions either immediately or subsequently for the duration of follow-up (range 4-24 months from first infusion, median $=13$ months). One patient (patient 1) had significant improvement in prior neurological deficits including improved gait, decreased dysmetria, and decreased nystagmus over the course of treatment. One patient (patient 4) had intermittent grade 3 abdominal pain which occurred during 4 of 12 cycles and lasted up to 2 days after the completion of these cycles. He underwent extensive work-up including abdominal plain X-rays, CT scan, endoscopy, and colonoscopy, all of which were negative. Because this patient had a ventriculoperitoneal shunt, it is possible that irritation of the peritoneum was caused by methotrexate traveling down the shunt. Two additional patients with ventriculoperitoneal shunts did not have abdominal pain at any time during the study. Additional grade 1 and 2 adverse events, either unrelated or possibly related to infusions, included headache, diarrhea, dry eye, nasal congestion, cough, gastroesophageal reflux, abdominal pain, sore throat, dysphagia, malaise, fatigue, fever, vomiting, anorexia, chills, back pain, dizziness, and oral mucositis. None of these adverse events were associated with any long-term problem over the duration of follow-up.

Six patients underwent baseline neuropsychological evaluations prior to the planned first cycle. Four of the five patients who received methotrexate infusions underwent follow-up neuropsychological evaluations after the third cycle. In all four patients, there were no significant changes from baseline tests assessing development and adaptive functioning, intelligence, memory, executive functioning, attention, expressive language, or visual and motor function. The fifth patient (patient 7) was removed from the protocol due to disease progression after the third cycle prior to obtaining repeat neuropsychological testing.

The median serum methotrexate level serum methotrexate level was $0.04 \mu \mathrm{mol} / \mathrm{L}$, and the range was $0.02-0.13 \mu \mathrm{mol} / \mathrm{L}$. No patient required leucovorin rescue. Median CSF trough methotrexate level was $3.18 \mu \mathrm{mol} / \mathrm{L}$, and the range was 0.53-212.36 $\mu \mathrm{mol} / \mathrm{L}$. Additional CSF studies for gram stain and culture, cell count, glucose, and protein did not reveal evidence of CSF infection in any patient.

In all five patients who received methotrexate infusions, MRI scans performed after the first cycle, third cycle, and every third cycle thereafter revealed no radiographic evidence of leukoencephalopathy or any damage to the brainstem, cerebellum, or cerebral cortex. Four patients underwent methotrexate cycles without interruption. Despite stable disease, intraventricular methotrexate infusions were stopped in patient 2 after cycle 3 at the request of the patient's family. Three months later, without further treatment, he had disease progression. With permission of the institutional review board, he underwent reoperation for tumor resection and was re-entered into the study. His postoperative MRI served as a new baseline prior to subsequent cycles.

Both patients with anaplastic ependymoma had progressive disease and were removed from the study after 9 and 3 cycles, respectively. Patient 2 died of progressive disease 20 months after enrollment, and patient 7 died of progressive disease 5 months after enrollment.

All three patients with medulloblastoma had decreased tumor burden after intraventricular methotrexate infusions. Two patients are currently alive (19 and 17 months after enrollment), and one (patient 1) died 25 months after enrollment. At the time of enrollment, patient 1 had massive tumor burden filling the fourth ventricle, coating the cerebellar folia, and involving the pituitary region, lateral ventricles, third ventricles, and thalamus. His disease burden progressively decreased with treatment but never reached the $50 \%$ threshold required for the definition of partial response (Fig. 3a). After cycle 12, his overall disease burden had decreased by $42 \%$. After cycle 15 , he was noted to have slight $(<25 \%)$ increase in 3 of 4 target lesions but complete disappearance of the 4 th target lesion (Fig. 3b). After cycle 18, one of his target lesions progressed by more than $25 \%$ so he met the definition of progressive disease and was removed from the study.

Patient 4 had disease only involving the spinal subarachnoid space. He had a partial response after cycle 3 that was maintained after cycles 6,9 , and 12 . After cycle 12 , only a thin line of enhancement remained at the site of a thoracic spine metastasis, and the decision was made to stop therapy. Unfortunately, his thoracic disease progressed on a follow-up MRI scan 3 months after he was removed from the protocol.

Patient 5 had metastatic nodules in the frontal horns of both lateral ventricles. Both nodules progressively decreased in size until, after cycle 12, she met the definition of partial response. Both lesions have continued to decrease in size and have nearly disappeared, and she still meets the definition of partial response after 18 cycles (Fig. 3c). She has been removed from the study but continues to receive intraventricular methotrexate into her fourth ventricle catheter off protocol at her home institution.

Patient 1 had positive CSF cytology in his baseline lumbar puncture and again after the first cycle. Cytology was negative for all subsequent lumbar puncture samples (after cycles 2, 3, 6, 12, 15, and 18) with one exception 
a

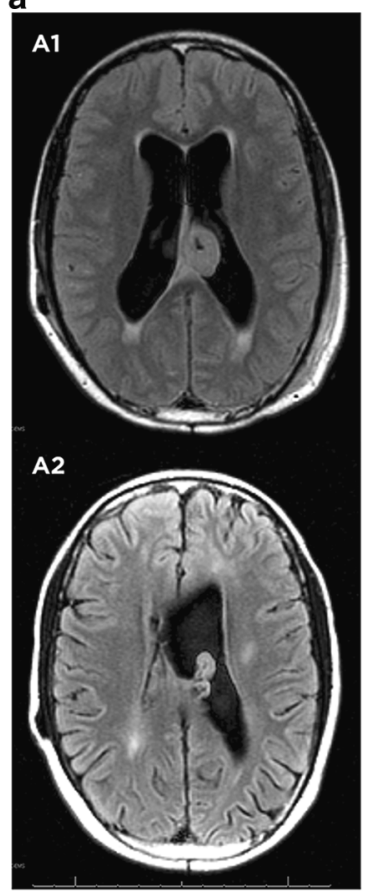

b


Fig. 3 MRI scans demonstrating treatment response in patients 1 and 4. a Fluid attenuated inversion recovery (FLAIR) MRI scans in patient 1 at baseline (top row, images $A 1, B 1$, and $C 1$ ) and after 15 cycles of intraventricular methotrexate (bottom row, figures $A 2, B 2$, and $C 2$ ), 10 months after initiation of the first cycle. There has been a considerable decrease in disease burden in the lateral ventricles, cerebellar folia, fourth ventricle, and cerebellopontine angles. FLAIR sequences are demonstrated because the majority of the tumor burden in the brain was non-enhancing and best visualized on FLAIR. b T1-

(after cycle 9). During his first three cycles, all $12 \mathrm{CSF}$ cytology samples from the fourth ventricle $(100 \%)$ were positive. Over cycles 4 through 12 , only 7 of 36 CSF samples from the fourth ventricle $(19.4 \%)$ were positive. From cycles 13 through 18, all 24 CSF samples from the fourth ventricle were negative. The remaining 4 patients who received treatment had negative cytology in all CSF samples from lumbar punctures and from the fourth ventricle.


weighted MRI of the spine with gadolinium in patient 4 at baseline (left, image $3 B 1$ ) and after 15 cycles of intraventricular methotrexate (right, image 3B2). Two small metastatic spine lesions (demonstrated by arrows) have disappeared. c Axial FLAIR sequences in patient 5 at baseline (image $3 C 1$ ) and after 18 cycles (image $3 C 2$ ) of intraventricular methotrexate. FLAIR sequences are demonstrated because both were non-enhancing lesions best visualized on FLAIR. There has been a considerable decrease in the lesions, both of which have nearly resolved

\section{Discussion}

For children with malignant tumors originating within the fourth ventricle, recurrences most commonly occur at the initial site of disease and/or in the subarachnoid spaces of the brain and spinal cord [4-6]. Ideally, novel treatment approaches should achieve both local and regional tumor control while minimizing systemic drug exposure, as metastatic disease outside of the central nervous system 
(CNS) is rare. Previous studies in piglets and non-human primates demonstrated that a catheter can be safely placed into the fourth ventricle and that chemotherapy can be infused without causing new neurological deficits or other recognized toxicity [7-10]. These infusions yielded high drug levels in the fourth ventricle as well the spinal subarachnoid space that are maintained for at least $24 \mathrm{~h}$ with undetectable or negligible systemic drug levels [7-10].

There are many potential advantages of fourth ventricular chemotherapy administration over infusions via repeated lumbar puncture or VAD placed into the lateral ventricle, both of which have been extensively utilized in previous studies [11-16]. Lumbar punctures are technically difficult in some patients and require sedation in young children. Moreover, infusions via lumbar puncture yield lower and less consistent ventricular drug levels than intraventricular infusions [17, 18]. While catheter placement into the lateral ventricle requires a separate surgical procedure from posterior fossa tumor resection, catheter placement into the fourth ventricle can be performed simultaneously to tumor resection and thereby spare patients an additional operation. Moreover, because lateral ventricle catheters are placed blindly through cerebral cortex, both catheter malposition and cerebral hemorrhage are reported complications [12, 19-21]. Fourth ventricle catheters are placed under direct vision without passing through brain parenchyma, so catheter malposition and hemorrhage are unlikely. Additionally, because lateral ventricle catheters are placed blindly into a ventricle that may be relatively small, some catheter holes may be within the ventricle and others within the cerebral cortex. Infusions may force the infused agent into brain parenchyma, contributing to leukoencephalopathy [12, 22-25]. In contrast, with fourth ventricular catheters, placement of the catheter under direct vision ensures that no catheter holes are within brain parenchyma. When placing fourth ventricular catheters and ventricular access devices, great care must be taken to secure the catheter in a way that ensures that it stays oriented parallel to the long access of the fourth ventricle to avoid brainstem injury. The catheter must be secured well to bone or fascia to ensure that it does not migrate.

It is important to note that many of the complications of lateral ventricle catheters described above can be minimized by using frameless stereotaxy to ensure that a catheter placed into the lateral ventricle is in the ideal position at the foramen of Monro. Moreover, fourth ventricle ventricular access devices may not be the ideal way to distribute drugs to metastatic lesions in certain patients depending on the anatomic location of the lesions. For example, some patients may potentially achieve higher drug levels in the lateral and third ventricles with a lateral ventricle catheter than with a fourth ventricle catheter.
In this study, serum methotrexate level $4 \mathrm{~h}$ after infusions was consistently low (median $0.04 \mu \mathrm{mol} / \mathrm{L}$ ). Low systemic drug levels are favorable when treating malignant brain tumors that only rarely spread outside of the central nervous system (CNS), as high systemic drug levels can cause toxicity to organs unaffected by cancer. In order to avoid additional reservoir taps besides those required for treatment, only trough CSF methotrexate levels were measured in this study. Trough CSF methotrexate levels were nearly 80 -fold higher (median $3.18 \mu \mathrm{mol} / \mathrm{L}$ ) than serum levels. It would be expected based upon prior animal studies that peak methotrexate levels, which were not measured in the current study, would likely have been several orders of magnitude higher than trough levels [7, 9, 10]. Ideally, methotrexate distribution would be assessed by lumbar spine CSF, but these were not performed to avoid lumbar punctures that would not have affected the patients' plan of care.

The primary objective of this study was to demonstrate that a chemotherapeutic agent may be infused via an implanted catheter into the fourth ventricle, next to the brainstem and cerebellum, without causing neurological toxicity. This objective was achieved based upon both radiographic and clinical criteria. Radiographically, no patient demonstrated leukoencephalopathy or other evidence of brain injury. Clinically, 240 consecutive infusions of methotrexate were administered in five patients without any new neurological deficits or other serious systemic toxicity. The patients who received the most infusions, patients 1 and 5, received 18 cycles (64 infusions) over approximately 1 year without any toxicity radiographically or clinically. No patient had any changes in neuropsychological examinations after 3 cycles of chemotherapy. Of course, neuropsychological changes may take years to develop after treatment, and long-term neuropsychological outcomes were not assessed by this pilot study.

Response to treatment in all three patients with medulloblastoma was an unexpected positive finding, as this pilot study used a low dose $(2 \mathrm{mg})$ of methotrexate to minimize the risk of toxicity. The outcomes of these three patients demonstrate that infusions into the fourth ventricle can lead to treatment response not only in the posterior fossa but also rostral to the infusions (in the lateral ventricles) and caudal to the infusions (in the spinal subarachnoid spaces). Unfortunately, both patients with ependymoma exhibited progressive disease after 9 and 3 cycles, respectively, and were therefore removed from the study. Historically, ependymoma is less chemosensitive than many other malignant brain tumors, and we hypothesize that either methotrexate is not the ideal drug for these patients or that $2 \mathrm{mg}$ is not an adequate dose. The concept of local delivery for ependymoma remains appealing, as recurrent 
ependymoma is very difficult to treat effectively with currently available systemic chemotherapy regimens.

In summary, this pilot trial has demonstrated that lowdose methotrexate can be infused into the fourth ventricle in patients with recurrent, malignant tumors without causing recognized neurological deficits or other significant toxicity. Partial responses in several patients offer proof of principle that intraventricular chemotherapy may be effective in treating malignant brain tumors, as the overwhelming majority of previous trials utilized simultaneous systemic chemotherapy. This trial will form the basis for future trials to explore the safety and efficacy of higher doses of methotrexate, as well as infusion of additional agents into the fourth ventricle, in an effort to expand treatment options and improve outcomes for patients with malignant posterior fossa tumors.

Acknowledgments This study has been supported by funding from Texas 4000 for Cancer and funds provided by the Division of Pediatrics at the University of Texas MD Anderson Cancer Center. The authors would like to thank Andy Dearwater for creating the illustration in Fig. 1 and to Allyson Lack for her assistance with image formatting. The authors additionally are grateful to Judy Moyes, M.D., and Noah's Light Foundation for supporting this project. Above all, we would like to thank the brave patients who enrolled in this trial and their wonderful families. Both MD Anderson Cancer Center and Dr. Laurence Cooper have a financial interest in ZIOPHARM Oncology, Inc., and Intrexon Corporation. On May 7, 2015, after completion of this study, Dr. Cooper was appointed as the Chief Executive Officer at ZIOPHARM. Dr. Cooper is now a Visiting Scientist at MD Anderson. This study had no relationship with Ziopharm Oncology Inc. or Intrexon.

Open Access This article is distributed under the terms of the Creative Commons Attribution 4.0 International License (http://crea tivecommons.org/licenses/by/4.0/), which permits unrestricted use, distribution, and reproduction in any medium, provided you give appropriate credit to the original author(s) and the source, provide a link to the Creative Commons license, and indicate if changes were made.

\section{References}

1. Balter-Seri J, Mor C, Shuper A, Zaizov R, Cohen IJ (1997) Cure of recurrent medulloblastoma: the contribution of surgical resection at relapse. Cancer 79(6):1241-1247

2. Lansky SB, List MA, Lansky LL, Ritter-Sterr C, Miller DR (1987) The measurement of performance in childhood cancer patients. Cancer 60(7):1651-1656

3. Juveland SL, Tyner JD (1995) Reagents and methods for the detection of methotrexate. In: Abbott Laboratories, US Patent Classification WO1995016026

4. Suit HD, Westgate SJ (1986) Impact of improved local control on survival. Int J Radiat Oncol Biol Phys 12(4):453-458

5. Silverman CL, Simpson JR (1982) Cerebellar medulloblastoma: the importance of posterior fossa dose to survival and patterns of failure. Int J Radiat Oncol Biol Phys 8(11):1869-1876

6. Choux M, Lena G, Alfonsi S, Bernard JL, Clement R, Elbaze F, Gambarelli D, Hassoun J, Lerpiniere JP, Lucas C, Pinsard N,
Raybaud D, Rigaud F, Ruffo M, Tapounie E (1982) Medulloblastoma. Neurochirurgie 28(Suppl 1):1-229

7. Sandberg DI, Crandall KM, Koru-Sengul T, Padgett KR, Landrum J, Babino D, Petito CK, Solano J, Gonzalez-Brito M, Kuluz JW (2010) Pharmacokinetic analysis of etoposide distribution after administration directly into the fourth ventricle in a piglet model. J Neurooncol 97(1):25-32

8. Sandberg DI, Crandall KM, Petito CK, Padgett KR, Landrum J, Babino D, He D, Solano J, Gonzalez-Brito M, Kuluz JW (2008) Chemotherapy administration directly into the fourth ventricle in a new piglet model. J Neurosurg Pediatr 1(5):373-380

9. Sandberg DI, Peet MM, Johnson MD, Cole P, Koru-Sengul T, Luqman A, Buitrago JC, Alam F, Wilkerson JR, Crandall KM, Kuluz JW (2012) Chemotherapy administration directly into the fourth ventricle in a nonhuman primate model. J Neurosurg Pediatr 9(5):530-541

10. Sandberg DI, Solano J, Petito CK, Mian A, Mou C, Koru-Sengul T, Gonzalez-Brito M, Padgett KR, Luqman A, Buitrago JC (2010) Safety and pharmacokinetic analysis of methotrexate administered directly into the fourth ventricle in a piglet model. J Neurooncol 100(3):397-406

11. DeAngelis LM (1998) Current diagnosis and treatment of leptomeningeal metastasis. J Neurooncol 38(2-3):245-252

12. Sandberg DI, Bilsky MH, Souweidane MM, Bzdil J, Gutin PH (2000) Ommaya reservoirs for the treatment of leptomeningeal metastases. Neurosurgery 47(1):49-54

13. Rutkowski S, Bode U, Deinlein F, Ottensmeier H, WarmuthMetz M, Soerensen N, Graf N, Emser A, Pietsch T, Wolff JE, Kortmann RD, Kuehl J (2005) Treatment of early childhood medulloblastoma by postoperative chemotherapy alone. N Engl J Med 352(10):978-986

14. Slavc I, Schuller E, Czech T, Hainfellner JA, Seidl R, Dieckmann K (1998) Intrathecal mafosfamide therapy for pediatric brain tumors with meningeal dissemination. J Neurooncol 38(2-3):213-218

15. Slave I, Schuller E, Falger J, Gunes M, Pillwein K, Czech T, Dietrich W, Rossler K, Dieckmann K, Prayer D, Hainfellner J (2003) Feasibility of long-term intraventricular therapy with mafosfamide $(\mathrm{n}=26)$ and etoposide $(\mathrm{n}=11)$ : experience in 26 children with disseminated malignant brain tumors. J Neurooncol 64(3):239-247

16. Fleischhack G, Jaehde U, Bode U (2005) Pharmacokinetics following intraventricular administration of chemotherapy in patients with neoplastic meningitis. Clin Pharmacokinet 44(1): $1-31$

17. Shapiro WR, Young DF, Mehta BM (1975) Methotrexate: distribution in cerebrospinal fluid after intravenous, ventricular and lumbar injections. N Engl J Med 293(4):161-166

18. Balis FM, Blaney SM, McCully CL, Bacher JD, Murphy RF, Poplack DG (2000) Methotrexate distribution within the subarachnoid space after intraventricular and intravenous administration. Cancer Chemother Pharmacol 45(3):259-264

19. Jacobs A, Clifford P, Kay HE (1981) The Ommaya reservoir in chemotherapy for malignant disease in the CNS. Clin Oncol 7(2):123-129

20. Obbens EA, Leavens ME, Beal JW, Lee YY (1985) Ommaya reservoirs in 387 cancer patients: a 15-year experience. Neurology 35(9):1274-1278

21. Shapiro WR, Posner JB, Ushio Y, Chemik NL, Young DF (1977) Treatment of meningeal neoplasms. Cancer Treat Rep 61(4):733-743

22. Rubinstein LJ, Herman MM, Long TF, Wilbur JR (1975) Disseminated necrotizing leukoencephalopathy: a complication of treated central nervous system leukemia and lymphoma. Cancer 35(2):291-305

23. Macdonald DR (1991) Neurologic complications of chemotherapy. Neurol Clin 9(4):955-967 
24. Chamberlain MC, Kormanik PA, Barba D (1997) Complications associated with intraventricular chemotherapy in patients with leptomeningeal metastases. J Neurosurg 87(5):694-699

25. Bleyer WA, Pizzo PA, Spence AM, Platt WD, Benjamin DR, Kolins CJ, Poplack DG (1978) The Ommaya reservoir: newly recognized complications and recommendations for insertion and use. Cancer 41(6):2431-2437 